\title{
Optimal Lehmer Mean Bounds for the Combinations of Identric and Logarithmic Means
}

\author{
Xu-Hui Shen, ${ }^{1}$ Wei-Ming Gong, ${ }^{2}$ and Yu-Ming Chu ${ }^{2}$ \\ ${ }^{1}$ College of Nursing, Huzhou Teachers College, Huzhou 313000, China \\ ${ }^{2}$ School of Mathematics and Computation Science, Hunan City University, Yiyang 413000, China \\ Correspondence should be addressed to Yu-Ming Chu; chuyuming2005@126.com
}

Received 11 July 2013; Accepted 5 August 2013

Academic Editors: M. Coppens, Y. Miao, and P.-y. Nie

Copyright (c) $2013 \mathrm{Xu}$-Hui Shen et al. This is an open access article distributed under the Creative Commons Attribution License, which permits unrestricted use, distribution, and reproduction in any medium, provided the original work is properly cited.

For any $\alpha \in(0,1)$, we answer the questions: what are the greatest values $p$ and $\lambda$ and the least values $q$ and $\mu$, such that the inequalities $L_{p}(a, b)<I^{\alpha}(a, b) L^{1-\alpha}(a, b)<L_{q}(a, b)$ and $L_{\lambda}(a, b)<\alpha I(a, b)+(1-\alpha) L(a, b)<L_{\mu}(a, b)$ hold for all $a, b>0$ with $a \neq b$ ? Here, $I(a, b), L(a, b)$, and $L_{p}(a, b)$ denote the identric, logarithmic, and $p$ th Lehmer means of two positive numbers $a$ and $b$, respectively.

\section{Introduction}

For $a, b>0$ with $a \neq b$, the logarithmic mean $L(a, b)$ and identric mean $I(a, b)$ are defined by

$$
\begin{aligned}
& L(a, b)=\frac{a-b}{\log a-\log b}, \\
& I(a, b)=\frac{1}{e}\left(\frac{b^{b}}{a^{a}}\right)^{1 /(b-a)},
\end{aligned}
$$

respectively. In the recent past, both mean values have been the subject of intensive research. In particular, many remarkable inequalities for $L$ and $I$ can be found in the literature [1-19]. In [14, 17, 20], inequalities between $L, I$, and the classical arithmetic-geometric mean of Gauss are proved. The ratio of identric means leads to the weighted geometric mean

$$
\frac{I\left(a^{2}, b^{2}\right)}{I(a, b)}=\left(a^{a} b^{b}\right)^{1 /(a+b)}
$$

which has been investigated in [11, 13, 21]. It might be surprising that the logarithmic mean has applications in physics, economics, and even in meteorology [22-24]. In [22], the authors study a variant of Jensen's functional equation involving $L$, which appears in a heat conduction problem. A representation of $L$ as an infinite product and an iterative algorithm for computing the logarithmic mean as the common limit of two sequences of special geometric and arithmetic means are given in [5]. In [25, 26], it is shown that $L$ can be expressed in terms of Gauss' hypergeometric function ${ }_{2} F_{1}$. And in [26], the authors prove that the reciprocal of the logarithmic mean is strictly totally positive; that is, every $n \times n$ determinant with elements $1 / L\left(a_{i}, b_{j}\right)$, where $0<a_{1}<a_{2}<\cdots<a_{n}$ and $0<b_{1}<b_{2}<\cdots<b_{n}$, is positive for all $n \geq 1$.

For $p \in \mathbb{R}$, the power mean $M_{p}(a, b)$ and Lehmer mean $L_{p}(a, b)$ of order $p$ of two positive numbers $a$ and $b$ with $a \neq b$ are defined by

$$
\begin{aligned}
M_{p}(a, b) & =\left(\frac{a^{p}+b^{p}}{2}\right)^{1 / p}, \\
L_{p}(a, b) & =\frac{a^{p+1}+b^{p+1}}{a^{p}+b^{p}} .
\end{aligned}
$$

It is well known that $M_{p}(a, b)$ and $L_{p}(a, b)$ are strictly increasing with respect to $p \in \mathbb{R}$ for fixed $a, b>0$ with $a \neq b$. The main properties for $M_{p}$ and $L_{p}$ are given in [27-32].

Let $A(a, b)=(a+b) / 2, G(a, b)=\sqrt{a b}$, and $H(a, b)=$ $2 a b /(a+b)$ be the arithmetic, geometric, and harmonic means 
of two positive numbers $a$ and $b$, respectively. Then it is well known that

$$
\begin{aligned}
\min \{a, b\} & <H(a, b)=L_{-1}(a, b) \\
& =M_{-1}(a, b)<G(a, b)=L_{-1 / 2}(a, b) \\
& =M_{0}(a, b)<L(a, b)<I(a, b)<A(a, b) \\
& =L_{0}(a, b)=M_{1}(a, b)<\max \{a, b\}
\end{aligned}
$$

for all $a, b>0$ with $a \neq b$.

The following sharp bounds for $L, I,(L I)^{1 / 2}$, and $(L+I) / 2$ in terms of power means are proved in $[2-4,6,8,9,33]$ :

$$
\begin{gathered}
M_{2 / 3}(a, b)<I(a, b)<M_{\log 2}(a, b), \\
M_{0}(a, b)<L(a, b)<M_{1 / 3}(a, b), \\
M_{0}(a, b)<\sqrt{I(a, b) L(a, b)}<M_{1 / 2}(a, b), \\
\frac{1}{2}(I(a, b)+L(a, b))<M_{1 / 2}(a, b)
\end{gathered}
$$

for all $a, b>0$ with $a \neq b$.

Alzer and Qiu [19] proved that

$$
\begin{aligned}
\alpha A(a, b)+(1-\alpha) G(a, b) & \\
<I(a, b) & <\beta A(a, b)+(1-\beta) G(a, b), \\
M_{p}(a, b) & <\frac{1}{2}(I(a, b)+L(a, b))
\end{aligned}
$$

for all $a, b>0$ with $a \neq b$ if and only if $\alpha \leq 2 / 3, \beta \geq 2 / e=$ $0.73575 \ldots$, and $p \leq \log 2 /(1+\log 2)=0.40938 \ldots$

The following sharp upper and lower Lehmer mean bounds for $L, I,(L I)^{1 / 2}$, and $(L+I) / 2$ are presented in [34]:

$$
\begin{gathered}
L_{-1 / 3}(a, b)<L(a, b)<L_{0}(a, b), \\
M_{-1 / 6}(a, b)<I(a, b)<L_{0}(a, b), \\
L_{-1 / 4}(a, b)<I^{1 / 2}(a, b) L^{1 / 2}(a, b)<L_{0}(a, b), \\
L_{-1 / 4}(a, b)<\frac{1}{2}(I(a, b)+L(a, b))<L_{0}(a, b)
\end{gathered}
$$

for all $a, b>0$ with $a \neq b$.

The purpose of this paper is to present the best possible upper and lower Lehmer mean bounds of the product $I^{\alpha}(a, b) L^{1-\alpha}(a, b)$ and the sum $\alpha I(a, b)+(1-\alpha) L(a, b)$ for any $\alpha \in(0,1)$ and all $a, b>0$ with $a \neq b$.

\section{Lemmas}

In order to establish our main result, we need several lemmas, which we present in this section.

Lemma 1. If $p \geq-1 / 3$, then the following statements are true:

(1) $18 p^{2}+21 p+8>0$;

(2) $270 p^{3}+567 p^{2}+384 p+104>0$;
(3) $1134 p^{4}+3429 p^{3}+3879 p^{2}+1950 p+448>0$;

(4) $1458 p^{5}+4941 p^{4}+6903 p^{3}+4809 p^{2}+1714 p+320>0$;

(5) $5346 p^{6}+18225 p^{5}+27297 p^{4}+21996 p^{3}+9672 p^{2}+$ $2264 p+320>0$

(6) $9 p^{3}+33 p^{2}+51 p+14>0$.

Proof. (1) We clearly see that

$$
\begin{aligned}
18 p^{2}+21 p+8 & >18 p^{2}+\left(-\frac{1}{3}\right) \times 21+8 \\
& =18 p^{2}+1>0
\end{aligned}
$$

(2) Let

$$
g_{1}(p)=270 p^{3}+567 p^{2}+384 p+104
$$

Then simple computations lead to

$$
\begin{aligned}
& g_{1}\left(-\frac{1}{3}\right)=29>0 \\
g_{1}^{\prime}(p)= & 6\left(135 p^{2}+189 p+64\right) \\
> & 6\left[135 p^{2}+189 \times\left(-\frac{1}{3}\right)+64\right] \\
= & 6\left(135 p^{2}+1\right)>0 .
\end{aligned}
$$

Therefore, Lemma 1(2) follows from (14) and (15).

(3) Let

$$
g_{2}(p)=1134 p^{4}+3429 p^{3}+3879 p^{2}+1950 p+448 \text {. }
$$

Then simple computations yield

$$
\begin{gathered}
g_{2}\left(-\frac{1}{3}\right)=116>0 \\
g_{2}^{\prime}(p)=4536 p^{3}+10287 p^{2}+7758 p+1950 \\
g_{2}^{\prime}\left(-\frac{1}{3}\right)=339>0 \\
g_{2}^{\prime \prime}(p)=13608 p^{2}+20574 p+7758 \\
>13608 p^{2}+20574 \times\left(-\frac{1}{3}\right)+7758 \\
=13608 p^{2}+900>0 .
\end{gathered}
$$

Therefore, Lemma 1(3) follows from (16) and (17). (4) Let

$$
\begin{aligned}
g_{3}(p)= & 1458 p^{5}+4941 p^{4}+6903 p^{3} \\
& +4809 p^{2}+1714 p+320 .
\end{aligned}
$$


Then simple computations lead to

$$
\begin{gathered}
g_{3}\left(-\frac{1}{3}\right)=\frac{247}{3}>0, \\
g_{3}^{\prime}(p)=7290 p^{4}+19764 p^{3}+20709 p^{2} \\
+9618 p+1714, \\
g_{3}^{\prime}\left(-\frac{1}{3}\right)=167>0, \\
g_{3}^{\prime \prime}(p)=29160 p^{3}+59292 p^{2}+41418 p+9618, \\
g_{3}^{\prime \prime}\left(-\frac{1}{3}\right)=1320>0, \\
g_{3}^{\prime \prime \prime}(p)=87480 p^{2}+118584 p+41418 \\
>87480 p^{2}+41418+118584 \times\left(-\frac{1}{3}\right) \\
=87480 p^{2}+1890>0 .
\end{gathered}
$$

Therefore, Lemma 1(4) follows from (18) and (19). (5) Let

$$
\begin{aligned}
g_{4}(p)= & 5346 p^{6}+18225 p^{5}+27297 p^{4}+21996 p^{3} \\
& +9672 p^{2}+2264 p+320 .
\end{aligned}
$$

Then simple computations yield

$$
\begin{gathered}
g_{4}\left(-\frac{1}{3}\right)=\frac{284}{3}>0 \\
g_{4}^{\prime}(p)=32076 p^{5}+91125 p^{4}+109188 p^{3} \\
+65988 p^{2}+19344 p+2264 \\
g_{4}^{\prime}\left(-\frac{1}{3}\right)=97>0 \\
g_{4}^{\prime \prime}(p)=160380 p^{4}+364500 p^{3} \\
+327564 p^{2}+131976 p+19344 \\
g_{4}^{\prime \prime}\left(-\frac{1}{3}\right)=228>0 \\
g_{4}^{\prime \prime \prime}(p)=108\left(5940 p^{3}+10125 p^{2}+6066 p+1222\right) \\
g_{4}^{\prime \prime \prime}\left(-\frac{1}{3}\right)=11340>0 \\
g_{4}^{(4)}(p)=1944\left(990 p^{2}+1125 p+337\right)
\end{gathered}
$$

It follows from (28) and the discriminant $\Delta=(1125)^{2}-$ $4 \times 990 \times 337=-68895<0$ of the quadratic function $f(p)=$ $990 p^{2}+1125 p+337$ that

$$
g_{4}^{(4)}(p)>0
$$

Therefore, Lemma 1(5) follows from (20)-(27) and (29).
(6) Let

$$
g_{5}(p)=9 p^{3}+33 p^{2}+51 p+14
$$

Then we have

$$
\begin{gathered}
g_{5}\left(-\frac{1}{3}\right)=\frac{1}{3}>0, \\
g_{5}^{\prime}(p)=27 p^{2}+66 p+51 \\
>27 p^{2}-66 \times\left(\frac{1}{3}\right)+51=27 p^{2}+29>0 .
\end{gathered}
$$

Therefore, Lemma 1(6) follows from (30) and (31).

Lemma 2. Suppose that $h(t)=(6 p+1) t^{6 p+6}-3 t^{6 p+4}-2(3 p+$ $2) t^{6 p+3}+(3 p+1) t^{3 p+6}-3 p t^{3 p+5}-3(p+1) t^{3 p+4}+3(p+1) t^{3 p+2}$ $+3 p t^{3 p+1}-(3 p+1) t^{3 p}+2(3 p+2) t^{3}+3 t^{2}-6 p-1$. If $p \in$ $(-1 / 3,-1 / 6)$, then $h(t)>0$ for $t>1$.

Proof. Let $h_{1}(t)=h^{\prime}(t) / t, h_{2}(t)=t^{4-3 p} h_{1}^{\prime \prime}(t), h_{3}(t)=h_{2}^{\prime \prime \prime}(t) / t$, and $h_{4}(t)=t^{4-3 p} h_{3}^{\prime \prime \prime}(t) /\left[9 p(p+1)(3 p+1)(3 p+2)^{2}(6 p+1)\right]$; then elaborated computations lead to

$$
h(1)=0 \text {, }
$$

$$
\begin{aligned}
& h_{1}(t)=6(p+1)(6 p+1) t^{6 p+4}-6(3 p+2) t^{6 p+2} \\
& -6(2 p+1)(3 p+2) t^{6 p+1} \\
& +3(p+2)(3 p+1) t^{3 p+4} \\
& -3 p(3 p+5) t^{3 p+3}-3(p+1)(3 p+4) t^{3 p+2} \\
& +3(p+1)(3 p+2) t^{3 p}+3 p(3 p+1) t^{3 p-1} \\
& -3 p(3 p+1) t^{3 p-2}+6(3 p+2) t+6, \\
& h_{1}(1)=0 \text {, } \\
& h_{1}^{\prime}(t)=12(p+1)(3 p+2)(6 p+1) t^{6 p+3} \\
& -12(3 p+1)(3 p+2) t^{6 p+1} \\
& -6(2 p+1)(3 p+2)(6 p+1) t^{6 p} \\
& +3(p+2)(3 p+1)(3 p+4) t^{3 p+3} \\
& -9 p(p+1)(3 p+5) t^{3 p+2} \\
& -3(p+1)(3 p+2)(3 p+4) t^{3 p+1} \\
& +9 p(p+1)(3 p+2) t^{3 p-1} \\
& +3 p(3 p-1)(3 p+1) t^{3 p-2} \\
& -3 p(3 p-2)(3 p+1) t^{3 p-3} \\
& +6(3 p+2) \text {, }
\end{aligned}
$$




$$
h_{1}^{\prime}(1)=0 \text {, }
$$

$$
\begin{aligned}
h_{2}(t)= & 36(p+1)(2 p+1)(3 p+2)(6 p+1) t^{3 p+6} \\
& -12(3 p+1)(3 p+2)(6 p+1) t^{3 p+4} \\
& -36 p(2 p+1)(3 p+2)(6 p+1) t^{3 p+3} \\
& +9(p+1)(p+2)(3 p+1)(3 p+4) t^{6} \\
& -9 p(p+1)(3 p+2)(3 p+5) t^{5} \\
& -3(p+1)(3 p+1)(3 p+2)(3 p+4) t^{4} \\
& +9 p(p+1)(3 p-1)(3 p+2) t^{2} \\
& +3 p(3 p-2)(3 p-1)(3 p+1) t \\
& -9 p(p-1)(3 p-2)(3 p+1), \\
& h_{2}(1)=12(3 p+1)\left(18 p^{2}+21 p+8\right),
\end{aligned}
$$

$$
h_{2}^{\prime}(t)=108(p+1)(p+2)(2 p+1)(3 p+2)(6 p+1) t^{3 p+5}
$$$$
-12(3 p+1)(3 p+2)(3 p+4)(6 p+1) t^{3 p+3}
$$$$
-108 p(p+1)(2 p+1)(3 p+2)(6 p+1) t^{3 p+2}
$$$$
+54(p+1)(p+2)(3 p+1)(3 p+4) t^{5}
$$$$
-45 p(p+1)(3 p+2)(3 p+5) t^{4}
$$$$
-12(p+1)(3 p+1)(3 p+2)(3 p+4) t^{3}
$$$$
+18 p(p+1)(3 p-1)(3 p+2) t
$$$$
+3 p(3 p-1)(3 p-2)(3 p+1) \text {, }
$$

$$
h_{2}^{\prime}(1)=12(3 p+1)(9 p+7)\left(18 p^{2}+21 p+8\right)
$$

$$
h_{2}^{\prime \prime}(t)=108(p+1)(p+2)(2 p+1)
$$$$
\times(3 p+2)(3 p+5)(6 p+1) t^{3 p+4}
$$$$
-36(p+1)(3 p+1)(3 p+2)
$$$$
\times(3 p+4)(6 p+1) t^{3 p+2}
$$$$
-108 p(p+1)(2 p+1)(3 p+2)^{2}(6 p+1) t^{3 p+1}
$$$$
+270(p+1)(p+2)(3 p+1)(3 p+4) t^{4}
$$$$
-180 p(p+1)(3 p+2)(3 p+5) t^{3}
$$$$
-36(p+1)(3 p+1)(3 p+2)(3 p+4) t^{2}
$$$$
+18 p(p+1)(3 p-1)(3 p+2),
$$

$h_{2}^{\prime \prime}(1)=36(3 p+1)(p+1)\left(270 p^{3}+567 p^{2}+384 p+104\right)$,

$$
\begin{aligned}
h_{3}(t)= & 108(p+1)(p+2)(2 p+1)(3 p+2) \\
& \times(3 p+4)(3 p+5)(6 p+1) t^{3 p+2} \\
& -36(p+1)(3 p+1)(3 p+2)^{2} \\
& \times(3 p+4)(6 p+1) t^{3 p} \\
& -108 p(p+1)(2 p+1)(3 p+1)(3 p+2)^{2} \\
& \times(6 p+1) t^{3 p-1}+1080(p+1)(p+2) \\
& \times(3 p+1)(3 p+4) t^{2} \\
& -540 p(p+1)(3 p+2)(3 p+5) t \\
& -72(p+1)(3 p+1)(3 p+2)(3 p+4) \\
& \times\left(1134 p^{4}+3429 p^{3}+3879 p^{2}+1950 p+448\right)
\end{aligned}
$$

$$
\begin{aligned}
h_{3}^{\prime}(t)= & 108(p+1)(p+2)(2 p+1)(3 p+2)^{2} \\
& \times(3 p+4)(3 p+5)(6 p+1) t^{3 p+1} \\
& -108 p(p+1)(3 p+1)(3 p+2)^{2} \\
& \times(3 p+4)(6 p+1) t^{3 p-1} \\
& -108 p(p+1)(2 p+1)(3 p-1)(3 p+1)(3 p+2)^{2} \\
& \times(6 p+1) t^{3 p-2}+2160(p+1)(p+2) \\
& \times(3 p+1)(3 p+4) t \\
& -540 p(p+1)(3 p+2)(3 p+5),
\end{aligned}
$$

$$
\begin{aligned}
& h_{3}^{\prime}(1)= 108(p+1)(3 p+1) \\
& \times\left(1458 p^{5}+4941 p^{4}+6903 p^{3}\right. \\
&\left.+4809 p^{2}+1714 p+320\right),
\end{aligned}
$$

$$
\begin{aligned}
h_{3}^{\prime \prime}(t)= & 108(p+1)(p+2)(2 p+1)(3 p+1) \\
& \times(3 p+2)^{2}(3 p+4)(3 p+5)(6 p+1) t^{3 p} \\
& -108 p(p+1)(3 p-1)(3 p+1)(3 p+2)^{2}(3 p+4) \\
& \times(6 p+1) t^{3 p-2}-108 p(p+1)(2 p+1)(3 p-2) \\
& \times(3 p-1)(3 p+1)(3 p+2)^{2}(6 p+1) t^{3 p-3} \\
& +2160(p+1)(p+2)(3 p+1)(3 p+4),
\end{aligned}
$$$$
h_{3}^{\prime \prime}(1)=108(p+1)(3 p+1)
$$

$$
\times\left(5346 p^{6}+18225 p^{5}+27297 p^{4}+21996 p^{3}\right.
$$$$
\left.+9672 p^{2}+2264 p+320\right)
$$ 


$$
\begin{aligned}
h_{4}(t)= & 36(p+2)(2 p+1)(3 p+4)(3 p+5) t^{3} \\
& -12(1-3 p)(2-3 p)(3 p+4) t \\
& +36(1-p)(1-3 p)(2-3 p)(2 p+1) \\
> & 36(p+2)(2 p+1)(3 p+4)(3 p+5) t \\
& -12(1-3 p)(2-3 p)(3 p+4) t \\
& +36(1-p)(1-3 p)(2-3 p)(2 p+1) \\
= & 24(3 p+4)\left(9 p^{3}+33 p^{2}+51 p+14\right) t \\
& +36(1-p)(1-3 p)(2-3 p)(2 p+1) .
\end{aligned}
$$

It follows from Lemma 1(6) and (49) that

$$
h_{4}(t)>0
$$

for $t>1$.

From Lemma 1(1)-(5) and (38)-(48), we clearly see that

$$
\begin{aligned}
& h_{2}(1)>0, \\
& h_{2}^{\prime}(1)>0, \\
& h_{2}^{\prime \prime}(1)>0, \\
& h_{3}(1)>0, \\
& h_{3}^{\prime}(1)>0, \\
& h_{3}^{\prime \prime}(1)>0 .
\end{aligned}
$$

Therefore, Lemma 2 follows from $9 p(p+1)(3 p+1)(3 p+$ $2)^{2}(6 p+1)>0$ and (32)-(36) together with (50) and (51).

Lemma 3. Inequality $\left[M_{2 / 3}(a, b)\right]^{\alpha}\left[L_{-1 / 3}(a, b)\right]^{1-\alpha}>L_{(\alpha-2) / 6}$ $(a, b)$ holds for any $\alpha \in(0,1)$ and all $a, b>0$ with $a \neq b$.

Proof. Without loss of generality, we assume that $a>b$. Let $t=(a / b)^{1 / 3}>1$ and $p=(\alpha-2) / 6$; then $p \in(-1 / 3,-1 / 6)$, $\alpha=6 p+2$, and from (1) and (4) we have

$$
\begin{gathered}
{\left[M_{2 / 3}(a, b)\right]^{\alpha}\left[L_{-1 / 3}(a, b)\right]^{1-\alpha}-L_{(\alpha-2) / 6}(a, b)} \\
=\left(b \left[\left(t^{3 p}+1\right)\left(t^{2}+1\right)^{3 p+2}(t+1)^{6 p+1}\right.\right. \\
\left.\left.-2^{9 p+3} t^{6 p+1}\left(t^{3 p+3}+1\right)\right]\right) \\
\times\left(2^{9 p+3} t^{6 p+1}\left(t^{3 p}+1\right)\right)^{-1} .
\end{gathered}
$$

Let

$$
\begin{aligned}
f(t)= & \log \left(t^{3 p}+1\right)+(3 p+2) \log \left(t^{2}+1\right) \\
& +(6 p+1) \log (t+1)-(6 p+1) \log t \\
& -\log \left(t^{3 p+3}+1\right)-3(3 p+1) \log 2 .
\end{aligned}
$$

Then simple computations lead to

$$
\begin{gathered}
f(1)=0, \\
f^{\prime}(t)=\frac{h(t)}{t(t+1)\left(t^{2}+1\right)\left(t^{3 p}+1\right)\left(t^{3 p+3}+1\right)},
\end{gathered}
$$

where $h(t)$ is defined as in Lemma 2.

From (54) and (55) together with Lemma 2, we clearly see that

$$
f(t)>0
$$

for $t>1$.

Therefore, Lemma 3 follows from (52) and (53) together with (56).

\section{Main Results}

Theorem 4. Inequality $L_{(\alpha-2) / 6}(a, b)<I^{\alpha}(a, b) L^{1-\alpha}(a, b)<$ $L_{0}(a, b)$ holds for any $\alpha \in(0,1)$ and all $a, b>0$ with $a \neq b$, and $L_{(\alpha-2) / 6}(a, b)$ and $L_{0}(a, b)$ are the best possible lower and upper Lehmer mean bounds for the product $I^{\alpha}(a, b) L^{1-\alpha}(a, b)$.

Proof. Inequality $I^{\alpha}(a, b) L^{1-\alpha}(a, b)<L_{0}(a, b)$ follows directly from (10) and (11).

For the other inequality, we note that

$$
\begin{aligned}
& I^{\alpha}(a, b) L^{1-\alpha}(a, b)-L_{(\alpha-2) / 6}(a, b) \\
& =I^{\alpha}(a, b) L^{1-\alpha}(a, b)-\left[M_{2 / 3}(a, b)\right]^{\alpha}\left[L_{-1 / 3}(a, b)\right]^{1-\alpha} \\
& \quad+\left[M_{2 / 3}(a, b)\right]^{\alpha}\left[L_{-1 / 3}(a, b)\right]^{1-\alpha}-L_{(\alpha-2) / 6}(a, b) .
\end{aligned}
$$

Therefore, $I^{\alpha}(a, b) L^{1-\alpha}(a, b)>L_{(\alpha-2) / 6}(a, b)$ follows from (7) and (10) together with Lemma 3 and (57).

Next, we prove that $L_{(\alpha-2) / 6}(a, b)$ and $L_{0}(a, b)$ are the best possible lower and upper Lehmer mean bounds for the product $I^{\alpha}(a, b) L^{1-\alpha}(a, b)$.

For any $0<\varepsilon<1$ and $x>0$, from (1) and (2) we have

$$
\begin{aligned}
& \lim _{x \rightarrow+\infty} \frac{I^{\alpha}(1, x) L^{1-\alpha}(1, x)}{L_{-\varepsilon}(1, x)} \\
& =\lim _{x \rightarrow+\infty}\left[\frac{x^{-\varepsilon}+1}{x^{\varepsilon-1}+1} e^{\alpha((\log x) /(x-1)-1)}\right. \\
& \left.\quad \times\left(1-\frac{1}{x}\right)^{1-\alpha} \frac{x^{\varepsilon}}{(\log x)^{1-\alpha}}\right]=+\infty, \\
& L_{(\alpha-2) / 6+\varepsilon}(1,1+x)-I^{\alpha}(1,1+x) L^{1-\alpha}(1,1+x) \\
& =\frac{(1+x)^{(\alpha+4) / 6+\varepsilon}+1}{(1+x)^{(\alpha-2) / 6+\varepsilon}+1}-e^{\alpha[(1+x) \log (1+x) / x-1]} \\
& \quad \times \frac{x^{1-\alpha}}{[\log (1+x)]^{\alpha}} .
\end{aligned}
$$


Letting $x \rightarrow 0$ and making use of Taylor expansion, one has

$$
\begin{aligned}
\frac{(1+x)^{(\alpha+4) / 6+\varepsilon}+1}{(1+x)^{(\alpha-2) / 6+\varepsilon}+1}-e^{\alpha[(1+x) \log (1+x) / x-1]} & \\
\quad & \times \frac{x^{1-\alpha}}{[\log (1+x)]^{\alpha}} \\
= & 1+\frac{1}{2} x+\left(\frac{\alpha}{24}-\frac{1}{12}+\frac{\varepsilon}{4}\right) x^{2}+o\left(x^{2}\right) \\
& -\left[1+\frac{\alpha}{2} x+\alpha\left(\frac{1}{8} \alpha-\frac{1}{6}\right) x^{2}+o\left(x^{2}\right)\right] \\
& \times\left[1+\left(\frac{1}{2}-\frac{\alpha}{2}\right) x+\left(\frac{1}{8} \alpha^{2}-\frac{1}{24} \alpha-\frac{1}{12}\right) x^{2}+o\left(x^{2}\right)\right] \\
= & 1+\frac{1}{2} x+\left(\frac{\alpha}{24}-\frac{1}{12}+\frac{\varepsilon}{4}\right) x^{2} \\
& -\left[1+\frac{1}{2} x+\left(\frac{\alpha}{24}-\frac{1}{12}\right) x^{2}\right]+o\left(x^{2}\right) \\
= & \frac{\varepsilon}{4} x^{2}+o\left(x^{2}\right) .
\end{aligned}
$$

Equations (58) and (59) imply that for any $0<\varepsilon<1$, there exist $X_{1}=X_{1}(\alpha, \varepsilon)>1$ and $\delta_{1}=\delta_{1}(\alpha, \varepsilon)>0$, such that $I^{\alpha}(1, x) L^{1-\alpha}(1, x)>L_{-\varepsilon}(1, x)$ for $x \in\left(X_{1},+\infty\right)$ and $L_{(\alpha-2) / 6+\varepsilon}(1,1+x)>I^{\alpha}(1,1+x) L^{1-\alpha}(1,1+x)$ for $x \in\left(0, \delta_{1}\right)$.

Theorem 5. Inequality $L_{(\alpha-2) / 6}(a, b)<\alpha I(a, b)+(1-$ $\alpha) L(a, b)<L_{0}(a, b)$ holds for any $\alpha \in(0,1)$ and all $a, b>0$ with $a \neq b$, and $L_{(\alpha-2) / 6}(a, b)$ and $L_{0}(a, b)$ are the best possible lower and upper Lehmer mean bounds for the sum $\alpha I(a, b)+$ $(1-\alpha) L(a, b)$.

Proof. Inequality $\alpha I(a, b)+(1-\alpha) L(a, b)<L_{0}(a, b)$ follows directly from (10) and (11), and inequality $L_{(\alpha-2) / 6}(a, b)<$ $\alpha I(a, b)+(1-\alpha) L(a, b)$ follows from Theorem 4 .

Next, we prove that $L_{(\alpha-2) / 6}(a, b)$ and $L_{0}(a, b)$ are the best possible lower and upper Lehmer mean bounds for the sum $\alpha I(a, b)+(1-\alpha) L(a, b)$.

For any $0<\varepsilon<1$ and $x>0$, from (1) and (2), we have

$$
\begin{aligned}
\lim _{x \rightarrow+\infty} \frac{\alpha I(1, x)+(1-\alpha) L(1, x)}{L_{-\varepsilon}(1, x)} \\
=\lim _{x \rightarrow+\infty}\left[\frac{x^{-\varepsilon}+1}{x^{\varepsilon-1}+1}\right. \\
\left.\quad \times\left(\alpha e^{(\log x) /(x-1)} x^{\varepsilon}+\frac{(1-\alpha)(1-1 / x)}{\log x} x^{\varepsilon}\right)\right]
\end{aligned}
$$$$
=+\infty \text {, }
$$

$$
\begin{aligned}
& L_{(\alpha-2) / 6+\varepsilon}(1,1+x)-\alpha I(1,1+x)-(1-\alpha) L(1,1+x) \\
& =\frac{(1+x)^{(\alpha+4) / 6+\varepsilon}+1}{(1+x)^{(\alpha-2) / 6+\varepsilon}+1}-\alpha e^{(1+x) \log (1+x) / x-1} \\
& \quad-\frac{(1-\alpha) x}{\log (1+x)}
\end{aligned}
$$

Letting $x \rightarrow 0$ and making use of Taylor expansion, one has

$$
\begin{aligned}
& \frac{(1+x)^{(\alpha+4) / 6+\varepsilon}+1}{(1+x)^{(\alpha-2) / 6+\varepsilon}+1}-\alpha e^{(1+x) \log (1+x) / x-1}-\frac{(1-\alpha) x}{\log (1+x)} \\
& =1+\frac{1}{2} x+\left(\frac{\alpha}{24}-\frac{1}{12}+\frac{\varepsilon}{4}\right) x^{2}+o\left(x^{2}\right) \\
& \quad-\alpha\left[1+\frac{1}{2} x-\frac{1}{24} x^{2}+o\left(x^{2}\right)\right] \\
& \quad-(1-\alpha)\left[1+\frac{1}{2} x-\frac{1}{12} x^{2}+o\left(x^{2}\right)\right] \\
& =\frac{\varepsilon}{4} x^{2}+o\left(x^{2}\right) .
\end{aligned}
$$

Equations (60)-(62) imply that for any $0<\varepsilon<1$, there exist $X_{2}=X_{2}(\alpha, \varepsilon)>1$ and $\delta_{2}=\delta_{2}(\alpha, \varepsilon)>0$, such that $\alpha I(1, x)+(1-\alpha) L(1, x)>L_{-\varepsilon}(1, x)$ for $x \in\left(X_{2},+\infty\right)$ and $L_{(\alpha-2) / 6+\varepsilon}(1,1+x)>\alpha I(1,1+x)+(1-\alpha) L(1,1+x)$ for $x \in\left(0, \delta_{2}\right)$.

\section{Acknowledgments}

This research was supported by the Natural Science Foundation of China under Grants 11071069 and 11171307 and the Natural Science Foundation of Zhejiang Province under Grants LY13H070004 and LY13A010004.

\section{References}

[1] G. Allasia, C. Giordano, and J. Pečarić, "On the arithmetic and logarithmic means with applications to Stirling's formula," Atti del Seminario Matematico e Fisico dell'Università di Modena, vol. 47, no. 2, pp. 441-445, 1999.

[2] H. Alzer, "Ungleichungen fur $(e / a)^{a}(b / e)^{b}$," Elemente der Mathematik, vol. 40, pp. 120-123, 1985.

[3] H. Alzer, "Ungleichungen für Mittelwerte," Archiv der Mathematik, vol. 47, no. 5, pp. 422-426, 1986.

[4] F. Burk, "The geometric, logarithmic and arithmetic mean inequality," The American Mathematical Monthly, vol. 94, no. 6, pp. 527-528, 1987.

[5] B. C. Carlson, "The logarithmic mean," The American Mathematical Monthly, vol. 79, no. 6, pp. 615-618, 1972.

[6] T. P. Lin, "The power mean and the logarithmic mesn," The American Mathematical Monthly, vol. 81, no. 8, pp. 879-883, 1974.

[7] J. Maloney, J. Heidel, and J. Pečarić, "A reverse Hölder type inequality for the logarithmic mean and generalizations," Journal of the Australian Mathematical Society Series B-Applied Mathematics, vol. 41, no. 3, pp. 401-409, 2000. 
[8] A. O. Pittenger, "Inequalities between arithmetic and logarithmic means," Univerzitet u Beogradu. Publikacije Elektrotehniv ckog Fakulteta. Serija Matematika, vol. 678-715, pp. 15-18, 1980.

[9] A. O. Pittenger, "The symmetric, logarithmic and power means," Univerzitet u Beogradu. Publikacije Elektrotehniv ckog Fakulteta. Serija Matematika, vol. 678-715, pp. 19-23, 1980.

[10] J. Sandor, "Inequalities for means," in Proceedings of the $3 \mathrm{rd}$ Symposium of Mathematics and its Applications (Timisoara, 1989), pp. 87-90, Romanian Academy, Timisoara, Romania, 1990.

[11] J. Sándor, "On the identric and logarithmic means," Aequationes Mathematicae, vol. 40, no. 1, pp. 261-270, 1990.

[12] J. Sándor, "A note on some inequalities for means," Archiv der Mathematik, vol. 56, no. 5, pp. 471-473, 1991.

[13] J. Sandor, "On certain identities for means," Studia Universitatis Babes-Bolyai Mathematica, vol. 38, no. 4, pp. 7-14, 1993.

[14] J. Sandor, "On certain inequalities for means," Journal of Mathematical Analysis and Applications, vol. 189, no. 2, pp. 602606, 1995.

[15] J. Sandor, "On refinements of certain inequalities for means," Archiv der Mathematik, vol. 31, no. 4, pp. 279-282, 1995.

[16] J. Sandor, “Two inequalities for means," International Journal of Mathematics and Mathematical Sciences, vol. 18, no. 3, pp. 621623, 1995.

[17] J. Sandor, “On certain inequalities for means II," Journal of Mathematical Analysis and Applications, vol. 199, no. 2, pp. 629635, 1996.

[18] J. Sándor, “On certain inequalities for means, III," Archiv der Mathematik, vol. 76, no. 1, pp. 34-40, 2001.

[19] H. Alzer and S.-L. Qiu, "Inequalites for means in two arivariables," Archiv der Mathematik, vol. 80, no. 2, pp. 201-215, 2003.

[20] M. K. Vamanamurthy and M. Vuorinen, "Inequalities for means," Journal of Mathematical Analysis and Applications, vol. 183, no. 1, pp. 155-166, 1994.

[21] J. Sandor and I. Rasa, "Inequalities for certain means in two arguments," Nieuw Archief voor Wiskunde, vol. 15, no. 41, pp. 5155, 1997.

[22] P. Kahlig and J. Matkowski, "Functional equations involving the logarithmic mean," ZAMM Zeitschrift fur Angewandte Mathematik und Mechanik, vol. 76, no. 7, pp. 385-390, 1996.

[23] A. O. Pittenger, "The logarithmic mean in n variables," American Mathematical Monthly, vol. 92, no. 2, pp. 99-104, 1985.

[24] G. Polya and G. Szego, Isoperimetric Inequalities in Mathematical Physics, Princeton University Press, Princeton, NJ, USA, 1951.

[25] B. C. Carlson, "Algorithms involving aritmetic and geometric means," American Mathematical Monthly, vol. 78, pp. 496-505, 1971.

[26] B. C. Carlson and J. L. Gustafson, "Total positivity of mean values and hypergeometric functions," SIAM Journal on Mathematical Analysis, vol. 14, no. 2, pp. 389-395, 1983.

[27] P. S. Bullen, D. S. Mitrinovic, and P. M. Vasic, Means and Their Inequalities, D. Reidel, Dordrecht, The Netherlands, 1988.

[28] K. B. Stolarsky, "Hölder means, Lehmer means, and $x^{-1} \log \cosh$ x." Journal of Mathematical Analysis and Applications, vol. 202, no. 3, pp. 810-818, 1996.

[29] Z. Liu, "Remark on inequalities between Hölder and Lehmer means," Journal of Mathematical Analysis and Applications, vol. 247, no. 1, pp. 309-313, 2000.
[30] E. F. Beckenbach, "A class of mean value functions," American Mathematical Monthly, vol. 57, pp. 1-6, 1950.

[31] E. F. Beckenbach and R. Bellman, Inequalities, Springer, New York, NY, USA, 1965.

[32] Z. Pales, "Inequalites for sums of powers," Journal of Mathematical Analysis and Applications, vol. 131, no. 1, pp. 265-270, 1988.

[33] K. B. Stolarsky, "The power and generalized logarithmic means," American Mathematical Monthly, vol. 87, no. 7, pp. 545-548, 1980.

[34] H. Alzer, "Bestmogliche Abschatzungen fur spezielle Mittelwerte," Prirodoslovno-Matematički Fakultet Sveučilišta u Zagrebu, vol. 23, no. 1, pp. 331-346, 1993. 


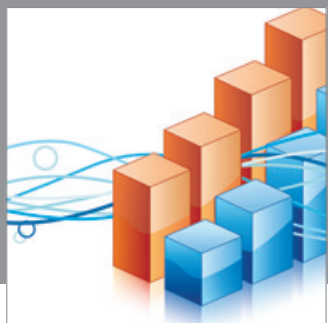

Advances in

Operations Research

mansans

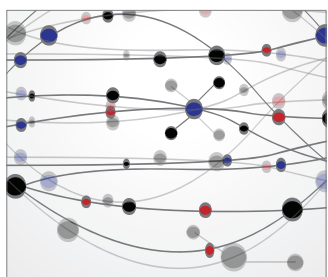

The Scientific World Journal
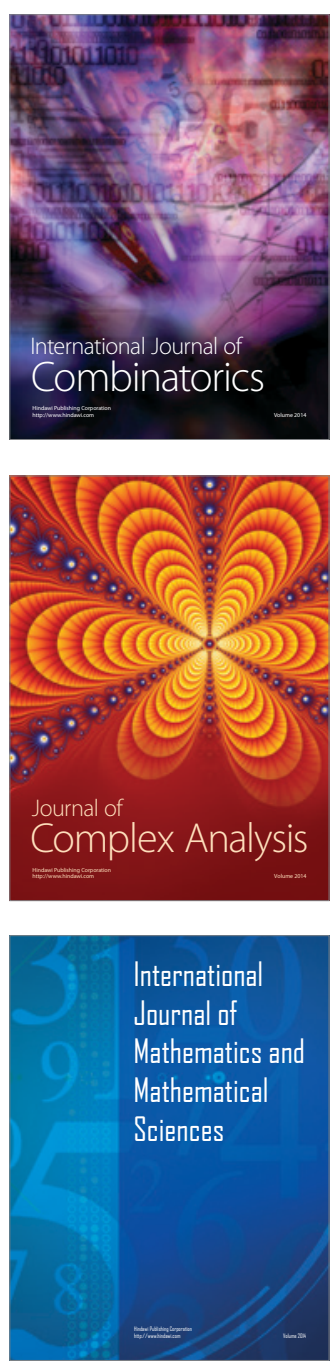
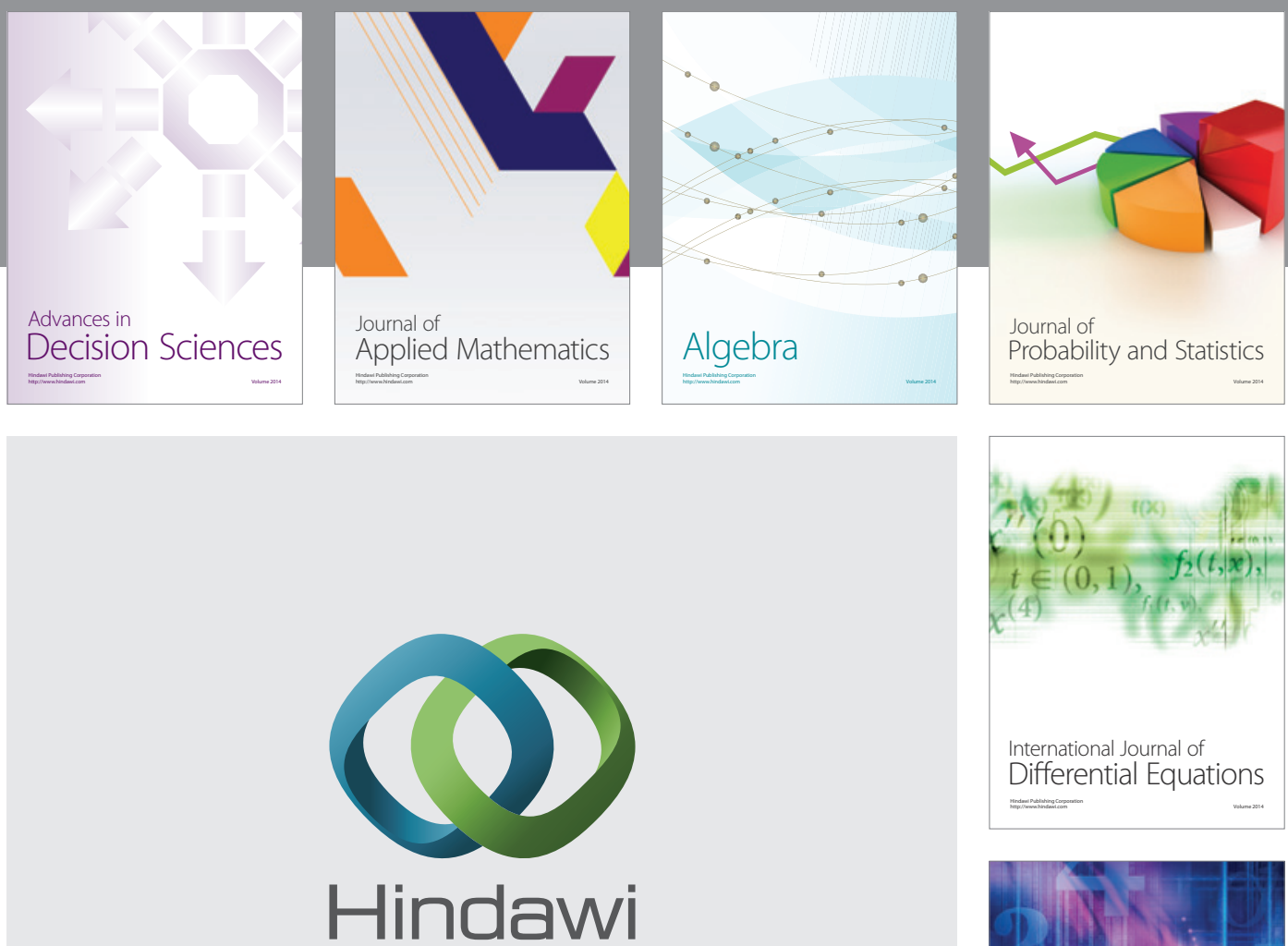

Submit your manuscripts at http://www.hindawi.com
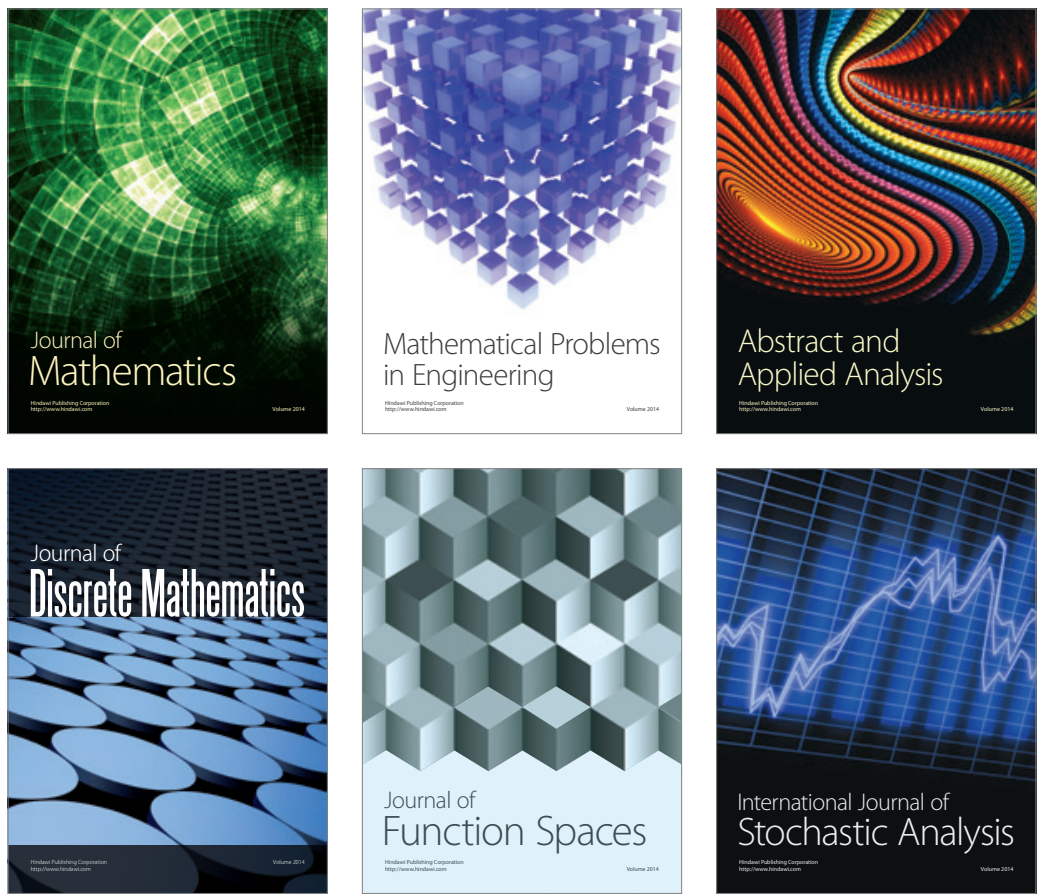

Journal of

Function Spaces

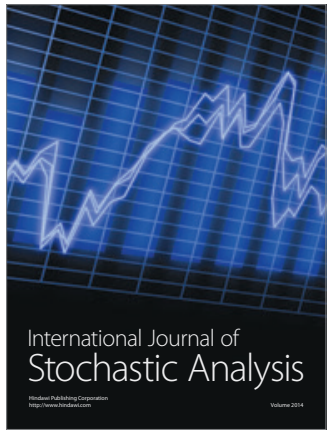

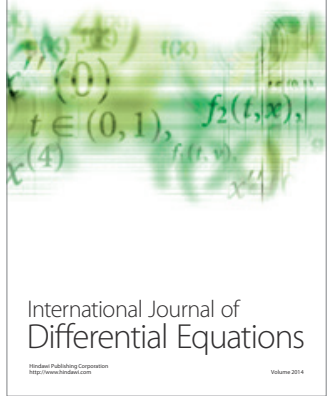
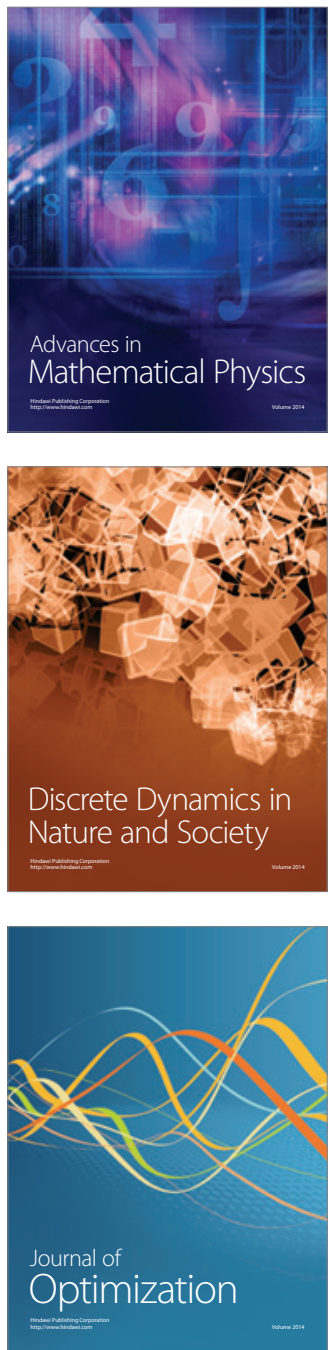